\title{
REALIDADE AUMENTADA PARA APRIMORAMENTO DE LABORATÓRIOS REMOTOS: UMA REVISÃO SISTEMÁTICA DA LITERATURA
}

\author{
Priscila Cadorin Nicolete, PPGIE, UFRGS, priscilanicolete@hotmail.com \\ Fabrício Herpich, PPGIE, UFRGS, fabricio_herpich@hotmail.com \\ Marta Adriana Machado da Silva, UNESC, marta.php@gmail.com \\ Liane Margarida Rockenbach Tarouco, PPGIE, UFRGS, liane@penta.ufrgs.br
}

\begin{abstract}
Resumo. Os Laboratórios Remotos de Realidade Aumentada configuram-se pela união de duas tecnologias já conhecidas e utilizadas na educação a fim de criar novos arranjos de práticas laboratoriais. Nesse contexto, este trabalho apresenta um estudo de mapeamento sistemático, por meio de uma análise bibliométrica e descritiva, com objetivo de compreender como ocorre essa união, identificar as tendências atuais, bem como os desafios e possíveis áreas futuras de pesquisa.
\end{abstract}

Palavras-chave: Laboratório Remoto, Realidade Aumentada, Tecnologia educacional

\section{AUGMENTED REALITY FOR THE IMPROVEMENT OF REMOTE LABORATORIES: A SYSTEMATIC LITERATURE REVIEW}

\begin{abstract}
The Augmented Reality Remote Labs are configured by the combination of two technologies already known and used in education in order to create new arrangements of laboratory practices. In this context, this work presents a systematic mapping study, through a bibliometric and descriptive analysis, in order to understand how this union occurs, to identify current trends, as well as challenges and possible future areas of research.
\end{abstract}

Keywords: Remote Labs, Augmented Reality, Educational Technology

\section{Introdução}

A evolução da tecnologia oferece novas possibilidades para o surgimento de diferentes formas de laboratórios práticos de ensino, tais como os Laboratórios Virtuais (LV) e Laboratórios Remotos (LR). Os laboratórios online têm se mostrado eficientes na superação de certas limitações dos laboratórios tradicionais, tais como altos custos, disponibilidade e custo de manutenção. Os LV são baseados em simulações, dos quais os alunos manipulam representações computacionais; já os LR permitem que os estudantes manipulem experimentos reais a distância, e esse acesso é realizado por meio de alguma interface que realiza a mediação entre o aluno e o equipamento.

Esses laboratórios são ótimas opções para as instituições de ensino que não possuem laboratórios presenciais ou que pretendem estender seus recursos, ou ainda que desejem compartilhar equipamentos com outras instituições. Além disso, o uso dos laboratórios online não está limitado a uma sala, do contrário, permanecem disponíveis sete dias por semana, 24 horas por dia, podendo ser explorado principalmente no ensino a distância.

A principal vantagem dos LR é a possibilidade de interação direta com equipamentos reais. A possibilidade de interagir com processos reais permite a descoberta de novos resultados, já que o usuário precisa calibrar os equipamentos com seus próprios dados (Cassini; Prattichizo, 2003). Ademais, o LR apresenta algumas limitações, tais como o feedback em fluxo de vídeo, que pode ser uma visualização simplificada e a falta de contato físico, não permitindo a total exploração da experiência.

Diante disso, surge uma nova modalidade: os Laboratórios Híbridos. Estes pretendem misturar elementos virtuais e remotos em um único espaço na tentativa de aproveitar as vantagens de cada um deles (Rodriguez-Gil et al., 2017). Nesse contexto, a tecnologia de 
Realidade Aumentada (RA) tem sido utilizada nesse novo formato de laboratório, a fim de criar uma experiência visual enriquecida para os usuários. A RA permite que objetos virtuais e objetos reais possam coexistir em um mesmo espaço. Desse modo, a combinação de RA com os LR pode auxiliar na superação das limitações destes, oferecendo uma visualização aumentada, não apenas mostrando em detalhes o que está ocorrendo no experimento, mas também possibilitando apresentar visualização ampliada, descrevendo o comportamento de aspectos internos das entidades do experimento (Rodriguez-Gil et al., 2017).

Nesse contexto, o estudo aqui exposto tem como objetivo compreender de que forma a RA é utilizada em Laboratórios Remotos para o aprimoramento das experiências online, identificando os tipos de experimentos desenvolvidos, as tecnologias utilizadas e os benefícios dessa união para as práticas educacionais, bem como são discutidos os desafios e áreas para futuras pesquisas.

Com isso tem-se a seguinte questão de pesquisa: "como a Realidade Aumentada é utilizada em Laboratórios Remotos em um contexto educacional e quais os avanços e desafios da área?". A fim de nortear a investigação algumas questões secundárias foram definidas: (i) que tipo de LR é desenvolvido com a ajuda da RA? E quais as principais vantagens dessa união?; (ii) quais as características das publicações sobre o tema? Autores, universidades e países que mais publicam, bem como a evolução temporal dos estudos; (iii) para quais níveis educacionais os laboratórios remotos de RA são desenvolvidos?; (iv) quais os principais enfoques dos estudos sobre RA em laboratórios remotos?, e; (v) quais foram as tecnologias mais utilizadas para desenvolver aplicações de RA em LR? Para responder tais questões, uma revisão sistemática da literatura e uma análise bibliométrica e descritiva do tema foram realizadas, assim como a identificação das principais implicações sobre o tema.

\section{Realidade Aumentada e Laboratórios online}

A Realidade Aumentada é o processo de sobreposição, no mundo real, de dados gerados por computador, ampliando assim o acesso à informação, gerando novas oportunidades de interação (Azuma, 1997). Essa sobreposição pode ser realizada por meio de marcadores que indicam o posicionamento em que os objetos virtuais devem aparecer em uma cena no mundo real. Para Azuma et al. (1997), uma aplicação de RA, além de combinar objetos reais e virtuais, deve alinhá-los entre si e devem ser executados de forma interativa e em tempo real.

A RA tem sido estudada desde meados dos anos 90, contudo tem ganhado destaque nos últimos anos, principalmente pela popularização dos dispositivos portáteis, que permitem o acesso simplificado a esse tipo de recurso. E é nesse contexto que estudos têm demonstrado o potencial da RA para a educação. Sahin e Yilmaz (2020) implementaram uma aplicação de RA para o ensino do Sistema Solar e o Universo para alunos do $7^{\circ}$ ano do ensino básico. Por meio de um livro interativo, os alunos tinham acesso a objetos 3D e vídeos que apresentavam detalhes das estrelas, constelações, cometas, planetas e unidades astronômicas. Segundo os autores, os alunos que utilizaram a RA apresentaram níveis mais elevados de desempenho e atitudes mais positivas em relação a disciplina do que aqueles que não utilizaram.

Martín-Gutiérrez et al. (2015) implementaram três aplicativos de RA a fim de contribuir para a autonomia dos estudantes em aulas práticas em laboratório. $\mathrm{O}$ primeiro aplicativo é um assistente que orienta o aluno nas tarefas em um laboratório prático, apresentando animações em $3 \mathrm{D}$, que são sobrepostas aos painéis práticos e indicam como os componentes (fios, bobinas, ímãs, etc) devem ser conectados. O segundo aplicativo visa auxiliar os alunos na leitura dos planos elétricos, ajudando na interpretação de símbolos complexos da área de Eletromecânica. Por fim, os autores apresentam um livro de RA com conteúdo teórico sobre princípios operacionais de máquinas elétricas.

Outros estudos destacam que a RA tem potencial para ser combinada com outras tecnologias, o que permite a exploração de novos arranjos de integração de TIC na educação. 
É o caso do laboratório remoto de RA, o qual pretende aprimorar a experimentação remota, unindo outras tecnologias que possam explorar todo o seu potencial, e consequentemente, reduzindo suas limitações. Surge, então, os Laboratórios Híbridos.

Nesse sentido, a RA pode ser utilizada para aumentar a sensação de realismo e melhorar a experiência do usuário em laboratórios remotos (Maiti; Maxwell; Kist, 2017; Mejías et al., 2013; Odeh; Shanab; Anabtawi, 2015). Nesses casos, a RA é operada a fim de adicionar módulos virtuais que podem interagir com hardwares dos LR de forma bidirecional. Conforme Rodriguez-Gil et al., (2017), RA permite que os estudantes experimentem novas sensações e explorem experiências de aprendizagem que, em alguns casos, podem exceder às oferecidas pelas aulas em laboratórios tradicionais. As possibilidades desse novo formato de laboratório serão apresentadas detalhadamente, na seção 4.

\section{Materiais e Métodos}

Esta pesquisa se caracteriza como exploratória, utilizando-se de procedimentos de revisão sistemática da literatura (RSL) em bases de dados eletrônicas, propondo-se a realizar análises bibliométrica e descritiva. Para determinar a confiabilidade da RSL quanto aos seus resultados e análises, bem como ser possível replicar seus procedimentos, foi utilizado o método Cochrane Handbook, com as suas fases de desenvolvimento: Formulação da pergunta; Seleção das bases de dados; Avaliação crítica dos estudos; Coleta de dados; Análise dos dados; Interpretação dos dados; Aprimoramento e atualização (Cochrane, 2019).

As variáveis identificadas foram "Realidade Aumentada" e "Laboratório Remoto", com Unidade de Observação: "aplicado a Educação". Optaram-se pelos bancos de dados Scopus, Portal de Periódico da CAPES, IEEE Xplore Digital Library e Biblioteca Digital Brasileira de Teses e Dissertações (BDTD).

Dessa forma, para fins de pesquisa na base de dados internacionais (Scopus e IEEE Xplore), foram selecionadas as seguintes palavras-chave, de forma hierárquica: "Augmented Reality", "Remote lab" e Education. Ao final, foram aplicados dois filtros de tipo de documentos: Article e Conference Paper. Para abranger um maior número de trabalhos, também foram realizadas pesquisas utilizando a palavras-chave: "Remote Experimen*", a fim de contemplar os trabalhos que utilizam a termologia Remote Experimentation e Remote Experiments para laboratórios remotos.

$\mathrm{Na}$ base de dados Scopus, foi primeiramente utilizado o termo "Augmented Reality". A busca realizada com tal palavra-chave ocorreu em: títulos, resumo, palavra-chave. Foram encontradas 27.385 publicações. Refinando a busca, utilizando a palavra-chave "Remote lab", retornaram 79 publicações e com a palavra-chave "Remote Experimen *", foram obtidas 42 publicações, totalizando 121 artigos (Figura 1).

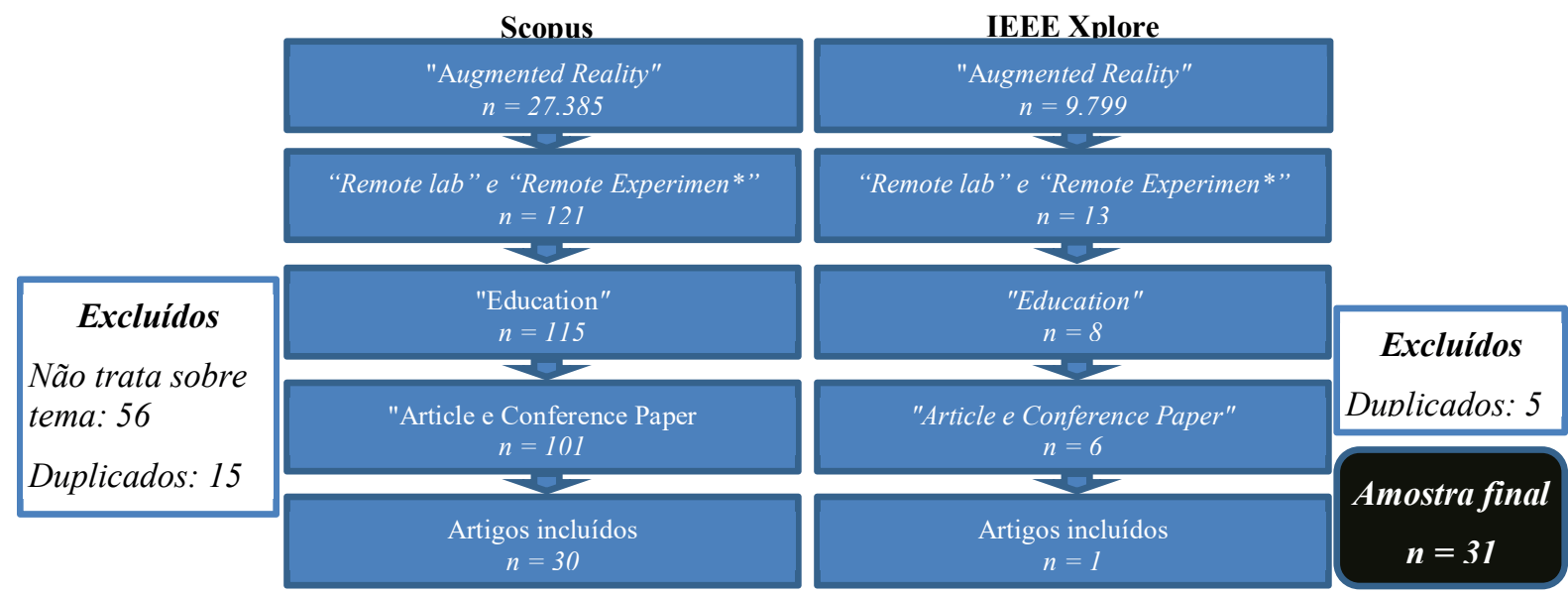

Figura 1: Resultados das buscas em cada base de dados - SCOPUS, IEEE Xplore Digital 
Com o filtro "Education" ficaram 115 publicações. Na sequência da aplicação dos filtros no sistema de busca, incluiu-se apenas Article e Conference Paper, eliminando os artigos em processo de publicação e revisões de conferência. Dessa forma, resultaram em 101 publicações (Figura 1).

Nesta etapa, realizou-se a leitura do título, resumo e introdução das 101 publicações encontradas. Dessas, 15 artigos foram excluídos por serem duplicados; outros 56 artigos foram excluídos por não tratarem do tema proposto, principalmente por apresentarem estudos apenas com o uso da RA, ou ainda trabalhos com estudo unicamente utilizando LR. Também foram excluídos os artigos que tratavam do uso de RA em Laboratórios hands-on, restando, portanto, 30 artigos. A Tabela 1 apresenta os critérios de inclusão e exclusão dos artigos para a análise.

Tabela 1: Critérios de exclusão e inclusão dos artigos para analise

\begin{tabular}{|l|l|}
\hline Critérios de exclusão & Critérios de inclusão \\
\hline Artigos que exploram apenas RA & Artigos que integram Realidade Aumentada em \\
Artigos que exploram apenas Laboratório Remoto & Laboratórios Remotos \\
Artigos que utiliza RA em Laboratórios hands-on & Artigos disponíveis em português, inglês ou espanhol \\
\hline
\end{tabular}

Na base de dados IEEE Xplore Digital, com o termo "Augmented Reality" foi encontrado 9.799 publicações. Com o refinamento da busca, utilizando a palavra-chave "Remote lab", o sistema retornou 5 publicações e com a palavra-chave "Remote Experimen*" o sistema retornou 4 publicações, totalizando 13 estudos. Aplicando o filtro com a palavra-chave "Education", ficaram 8 artigos. Desses, 5 foram excluídos da amostra final por já constarem na pesquisa realizada na base Scopus e 2 por não se tratar de artigo em periódicos ou de conferências, resultando, portanto, em apenas 1 publicação.

Foram realizadas ainda pesquisas na base de dados BDTD, para identificar trabalhos de teses e dissertações que contemplem o assunto e no Portal de Periódicos da CAPES. Utilizando o mesmo protocolo de busca, em ambas as bases, não foram obtidos trabalhos que integrassem Realidade Aumentada aos Laboratórios Remotos. Com isso, ao final das pesquisas, aplicação dos filtros e dos critérios de exclusão, foram selecionados para a análise 31 artigos, que configuram-se na amostra final do estudo.

\section{Resultados}

O primeiro registro encontrado sobre o tema é do ano de 2000, entretanto, nos próximos 10 anos ocorreu apenas uma publicação, em 2005. A partir de 2011, todos os anos apresentaram pesquisas na área, sendo que os anos de 2016 e 2017 registraram o maior número de publicações (Figura 2).

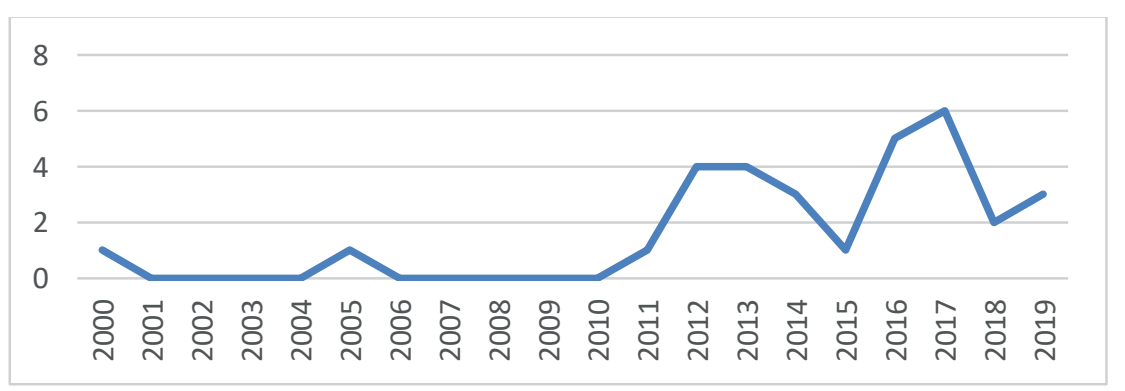

Figura 2: Publicações por ano

Os países que mais publicaram sobre o tema são a Espanha, com 10 trabalhos, representando 32,2\% dos trabalhos, seguida pela Austrália, com 7 trabalhos (22,5\%). Ao longo dos anos, os espanhóis e australianos têm se destacado em estudos que envolvem o uso 
de LR na educação (Dos Santos; Fernandes; Da Silva, 2017) (Figura 3). Vale destacar que o Brasil não apresentou estudos sobre o tema, o que justifica os resultados das pesquisas nas bases de dados nacionais.

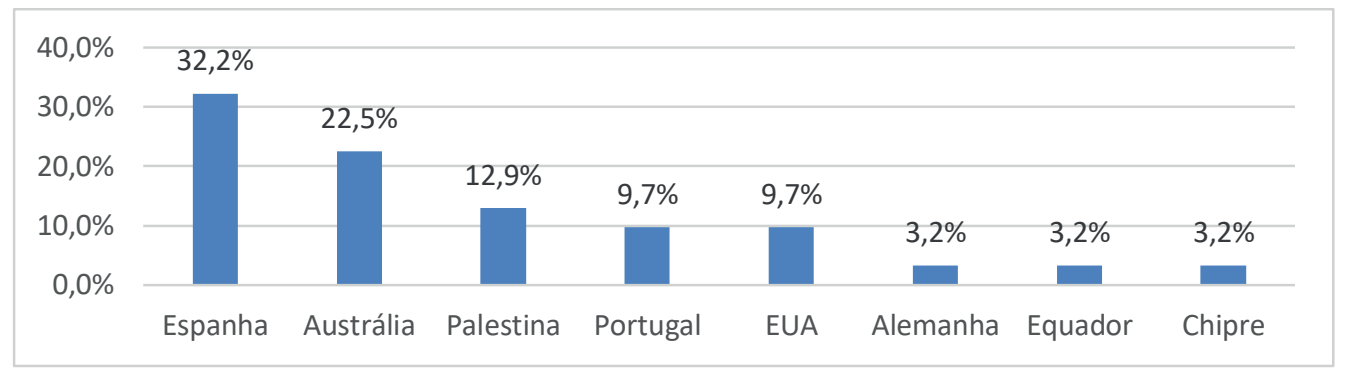

Figura 3: Percentual dos cinco países que mais publicaram sobre o tema

Também foi apurado para quais níveis de ensino são desenvolvidas as soluções de Laboratórios Remotos com RA. A maioria dos artigos, 64,5\%, relacionam-se a aplicações no ensino superior em cursos de graduação e $6,4 \%$ em cursos de pós-graduação, sendo o ensino de Engenharia o principal foco desses estudos. Em geral, os autores justificam que os cursos em engenharias necessitam da prática em laboratórios para a inserção do egresso no mercado de trabalho e que a experimentação é de fundamental importância para a aprendizagem da área. Vale destacar que nenhum dos estudos tem foco no ensino básico (fundamental ou médio). Ainda, foram encontrados estudos $(29,1 \%)$ que não informaram para qual nível de ensino o recurso foi desenvolvido.

A fim de conhecer como é utilizado a RA em Laboratórios Remotos na educação, foi realizada uma análise referente ao enfoque dos estudos, ou seja, sob qual ponto de vista os artigos foram desenvolvidos. Dessa forma, após a leitura dos artigos, foram identificadas as seguintes categorias:

- Descrição do Experimento: relaciona os artigos que descrevem o LR e/ou seus aspectos técnicos. Alguns discorrem sobre seus possíveis benefícios, porém não é apresentado a aplicação do laboratório em sala de aula.

- Avaliação Técnica do protótipo: nessa categoria fazem parte os artigos que, além de descrever o experimento, realizam uma avaliação técnica do protótipo.

- Avaliação da aceitação da ferramenta: aqui relacionam os artigos que, além da descrição do experimento, realizam uma avaliação da sua aceitação junto aos estudantes e/ou professores. Nesses estudos, de modo geral, aplicam questionários de satisfação e motivação ao utilizar os laboratórios remotos de RA; em alguns casos comparando com o uso de LR tradicionais e laboratórios hands-on.

- Aprendizagem: nesta categoria foram contabilizados os artigos que focaram na avaliação dos possíveis benefícios dos laboratórios remotos de RA para os processos de ensino e aprendizagem. Realizando algum tipo de análise sobre o desempenho de aprendizagem dos estudantes.

A Tabela 2 apresenta a frequência das publicações quanto ao seu enfoque, bem como relaciona os artigos de cada categoria.

Tabela 2: Frequência e publicações quanto ao seu enfoque

\begin{tabular}{|c|c|c|c|}
\hline Enfoque & Estudos & $\mathrm{n}$ & $\%$ \\
\hline $\begin{array}{l}\text { Descrição do } \\
\text { Experimento }\end{array}$ & $\begin{array}{l}\text { (Salzmann; Gillet; Huguenin, 2000); (Lo et al., 2012); (Mejías et al., 2013); } \\
\text { (Vargas et al., 2013); (Chaczko et al., 2014); (Guenaga et al., 2014); (Granado; } \\
\text { Abad; Palomeque, 2016) (Maiti; Kist; Smith, 2016); (Smith et al., 2016b); (Sun } \\
\text { et al., 2016); (Fabregas; Dormido-Canto; Dormido, 2017); (Maiti et al., 2017); } \\
\text { (Márquez et al., 2017); (Rodrigues et al., 2017); (Grodotzki; Ortelt; Tekkaya, } \\
\text { 2018); (Zandavi; Chung, 2018); (Smith et al., 2019). }\end{array}$ & 17 & $54,8 \%$ \\
\hline
\end{tabular}




\begin{tabular}{|l|l|l|l|}
\hline $\begin{array}{l}\text { Avaliação da } \\
\begin{array}{l}\text { aceitação da } \\
\text { ferramenta }\end{array}\end{array}$ & $\begin{array}{l}\text { (Andújar; Mejias; Marquez, 2011); (Mejías; Andújar, 2012); (Odeh et al., } \\
\text { (Cardoso et al., 2014); (Odeh et al., 2015); (Rodriguez-Gil et al., 2017); }\end{array}$ & $\mathbf{2 9 , 1 \%}$ \\
\hline $\begin{array}{l}\text { Rev. da literatura } \\
\text { (Lasica et al., 2016); (Lasica et al., 2017); (Arici et al., 2019). }\end{array}$ & & \\
\hline Avaliação Técnica & (Marín et al., 2005). & 1 & $\mathbf{3 , 2 \%} \%$ \\
\hline Aprendizagem & (Banky; Blicblau, 2019) & 1 & $\mathbf{3 , 2 \%}$ \\
\hline
\end{tabular}

Como pode ser observado, mais de 50\% dos artigos apresentam enfoque na descrição dos experimentos e/ou da sua infraestrutura e $29,1 \%$ dedicaram-se a avaliação da aceitação da ferramenta. O que chama a atenção é a baixa quantidade de estudos que preocuparam-se com a avaliação dos aspectos educacionais. Apenas um artigo realizou algum tipo de análise sobre o desempenho dos estudantes. Vale destacar que quase $50 \%$ das revistas que publicaram sobre o tema não possuem enfoque educacional, preocupando-se principalmente com os aspectos tecnológico das soluções apresentadas.

Ainda, foram encontradas três revisões sistemáticas e um estudo que realizou a avaliação técnica do protótipo. As RSL encontradas não têm como objetivo entender o uso da RA em laboratórios remotos como descrito no atual estudo, mas, sim, pretendem identificar o uso de diferentes laboratórios na educação.

Entre as principais tecnologias de RA utilizadas para aprimorar as experiências remotas estão o motor de jogo Unity3D, que permite o desenvolvimento de aplicativos com uso de imagens 3D e bibliotecas de rastreamento de marcadores como Vuforia. Para a criação de objetos 3D foram utilizados softwares como Blender3D e Coin3D (Tabela 3).

Tabela 3: Tipos de tecnologias para o desenvolvimento dos laboratórios híbridos

\begin{tabular}{|l|l|}
\hline Estudos & Tecnologias utilizadas \\
\hline Chaczko et al. (2014) & OpenCV \\
\hline Sun et al. (2016); Maiti, Maxwell \& Kist (2017) & Unity3D, Vuforia \\
\hline Rodrigues et al. (2017) & Unity3D, ARToolKit \\
\hline Rodriguez-Gil et al. (2017) & Unity3D, WebGL \\
\hline Andújar et al. (2011); Mejias \& Marquez (2012); Mejías \& Andújar (2013) & ARToolkit, OpenGL e Coin3D \\
\hline Mejías et al. (2013); Márquez et al. (2017); Fabregas, Canto, Dormido (2017) & Easy Java Simulations \\
\hline Maiti, Kist e Smith (2016); Smith et al (2016); & SNAP programming platform \\
\hline
\end{tabular}

Além disso, três estudos utilizam a ferramenta Easy Java Simulations (EJS), desenvolvida por Vargas et. al. (2013), da Universidade Nacional de Educação a Distância da Espanha, que permite a criação de experimentos virtuais e remotos de forma simplificada. Outra plataforma identificada entre os estudos é a SNAP, uma plataforma de programação por meio de linguagem de programação em blocos (Smith et al., 2016a).

\subsection{Realidade Aumentada e Laboratórios Remotos: as possibilidades dessa união}

No ano de 2000 uma abordagem unindo elementos de RA em laboratórios remotos já era apresentada pelos autores Salzmann, Gillet e Huguenin (2000). No estudo, o LR é usado para controlar um pêndulo invertido e os objetos virtuais utilizados são derivados das medições feitas no sistema real, que são combinados com a imagem de vídeo da câmera webcam do experimento real.

Em estudos mais recentes, um grupo de pesquisadores da Universidade de Huelva propõe o conceito de Laboratório Remoto Aumentado (LRA), que segundo Andújar et al., (2011), a RA é utilizada em LR para adicionar módulos virtuais que podem interagir com hardwares desses laboratórios de forma bidirecional (Andújar et al., 2011). Os autores desenvolveram um LRA que corresponde a um sistema de controle digital baseado em uma placa FPGA (Field Programmable Gate Array) para o ensino de automação, aplicado em cursos de ensino superior de engenharia. As placas FPGA são tradicionalmente utilizadas em 
aulas práticas, das quais os alunos precisam desenvolver sistemas para controlar periféricos de entrada e analisar as respostas em periféricos de saídas. Já com a utilização da RA, esses periféricos podem ser expandidos, criando complexos arranjos de componentes, simulando diferentes situações. No trabalho referido, os autores descrevem um tanque de água virtual controlável pela placa FPGA. Entretanto, segundo eles, por meio da RA, a mesma placa pode gerar cenários diferentes, utilizando diferentes composições de objetos virtuais.

Mejías et al. (2013) descrevem um LRA para programação de um PLC (Controlador Lógico Programável) a fim de automatizar uma planta de laboratório real, que é aumentada por objetos virtuais $3 \mathrm{D}$ que interagem com os reais. Por meio de um computador, o aluno pode operar o PLC remoto e observar a evolução do esquema real de acordo com a programação que ele enviou ao controlador. "O sistema RA converte a correia transportadora em uma instalação que deve colocar uma ficha e uma etiqueta na garrafa que é movida pela correia" [tradução nossa] (Márquez et al., 2017).

Maiti, Maxwell e Kist (2017) integraram RA em um Laboratório Remoto utilizando Interface de Usuário Natural (Natural User Interfaces - NUI) para a interação do usuário com o experimento. Por meio de uma NUI, é possível ler entradas do usuário no sistema sem utilizar dispositivos de entrada comuns, como teclado ou mouse. O experimento remoto desenvolvido permite a manipulação de engrenagens de um robô LEGO com a utilização de RA e NUI. Para os autores, com a utilização dessas tecnologias em LR, é possível proporcionar um alto grau de interatividade, oferecendo experiências remotas mais realistas, aproximando-se dos experimentos realizadas em laboratórios hands-on.

\section{Discussão dos resultados}

É indiscutível que aparatos experimentais constituem uma das importantes ferramentas para o ensino das disciplinas das áreas STEM em todos os níveis educacionais. Há anos, pesquisadores têm explorado diferentes formas de experimentação a fim de aprimorar essas práticas. Atualmente, há uma tendência clara para o uso extensivo de diferentes tipos de laboratórios: hands-on, virtuais e remotos. A combinação da RA e os laboratórios remotos surge desse paradigma, uma vez que pretende unir aspectos relevantes de cada um dos três tipos de laboratórios.

Por sua vez, a RA vem sendo estudada no campo educacional com o objetivo de proporcionar experiências tecnológicas de aprendizagem visualmente mais atraentes em diversas áreas do conhecimento, permitindo que conceitos ensinados em aulas tradicionais possam ser melhores compreendidos com o uso de multimídia interativa. Conforme Herpich (2019), a partir da sobreposição no mundo real de recursos virtuais como imagens, vídeos, textos e objetos 3D é possível criar soluções educacionais que contribuam na compreensão de fenômenos complexos. As principais vantagens do uso da RA para o ensino versam no fornecimento de informações contextualizadas, a visualização de fenômenos invisíveis e a interação com objetos 3D integrados no mundo real, favorecendo para a motivação e engajamento dos estudantes.

Nesse sentido, Maiti et al., (2017) afirmam que a RA pode ser aplicada em LR de duas formas: "visibilidade induzida": que é a capacidade de apresentar, por meio de RA, objetos reais que não podem ser visíveis pela câmera; e "informações sobrepostas", no qual é a apresentado informações extras, por exemplo em formato de textos, que estão relacionados a certos objetos reais, dessa forma os objetos e as informações sobrepostas devem ser atualizadas em tempo real para refletir a alteração no estado do objeto.

Diante disso, percebe-se que a disponibilização de laboratórios híbridos com uso da RA pode acarretar em aplicações sofisticadas, suprindo limitações dos LR, oferecendo visibilidade virtual a componentes reais, que por vezes são difíceis de ser apresentados em um streaming de vídeo comum aos LR. Ainda, permite incluir informações extras, a fim de 
representar algum elemento abstrato que seria impossível de verificar mesmo em laboratórios tradicionais. Com isso, um novo leque para a experimentação remota se apresenta, expandindo suas possibilidades e criando novas formas de experimentação.

Outro benefício gerado pelo uso de RA em laboratórios remotos é o reaproveitamento dos recursos físicos. Por meio da RA, um laboratório remoto pode ser expandido, reduzido ou modicado, fazendo com que um mesmo experimento seja usado para diferentes objetivos educacionais, sem a necessidade de modificar o ambiente físico (Mejías; Andújar, 2012; Rodriguez-Gil et al., 2017).

Entre as tecnologias mais utilizadas para o desenvolvimento dos laboratórios remotos de RA estão o motor de jogo Unity 3D e o framework Vuforia. O Unity 3D nativamente oferece suporte ao desenvolvimento para diferentes plataformas, o que facilita e flexibiliza o desenvolvimento das aplicações. O Vuforia oferece uma plataforma de visão computacional que permite a construção, de forma simplificada, de experiências interativas e apresenta diversos recursos relacionados à RA (Herpich; Guarese; Tarouco, 2017).

Entretanto, Rodriguez-Gil et al. (2017) destacam a importância de desenvolver uma arquitetura para os laboratórios remotos de RA totalmente baseada na web. Segundo os autores, a web é a tendência atual para o desenvolvimento de recursos digitais, permitindo que os laboratórios possam ser executados em qualquer plataforma que tenha um navegador compatível, sem a necessidade da instalação de novos aplicativos. As recomendações expressas no artigo de Rodriguez-Gil et al (2017) podem ser um bom caminho para pesquisadores que desejam implementar Laboratórios Híbridos.

Abaixo, são relacionadas algumas sugestões baseadas nos achados deste estudo:

- Ainda encontramos na literatura um número limitado de estudos que exploram técnicas de RA em laboratórios remotos. Mais estudos, com maior diversidade de métodos de pesquisas, são necessários para avaliar oportunidades desses recursos para o ensino.

- As pesquisas sobre o tema, até o momento, têm colocado seus esforços especialmente no desenvolvimento e avaliação da aceitação da ferramenta. Com isso, acredita-se que análise da eficácia educacional desses recursos é um tópico importante para futuras pesquisas, a fim de investigar se o uso de formas inovadoras de experimentação pode melhorar os resultados educacionais.

- Aspectos pedagógicos também precisam ser discutidos em futuras pesquisa. Quais abordagens pedagógicas são mais adequadas para a inclusão dos laboratórios remotos de RA na educação?

- A aprendizagem móvel é uma tendência no campo educacional, principalmente quando se trata do uso da RA. Entretanto, nos estudos encontrados os laboratórios desenvolvidos não levam em consideração esse aspecto.

- A maioria dos estudos sobre laboratórios remotos de RA são realizados no ensino superior, envolvendo, principalmente, cursos de engenharia. Nenhum estudo teve seu foco no ensino básico. Este pode ser um importante grupo amostral para estudos futuros. O Brasil ainda apresenta um ensino pautado em aulas excessivamente expositivas, principalmente pela falta de laboratórios de Ciências, esses recursos podem contribuir para a superação de tal carência.

- Sugere-se, também, a expansão das áreas de aplicação desses recursos no ensino superior. Em todos os cursos que envolvam as áreas STEM, aulas práticas de laboratório são de fundamental importância, pois permitem que os alunos experienciam o conhecimento teórico. Com o advento dos cursos em modalidade de ensino a distância (EAD), recursos tecnológicos inovadores se apresentam como uma fonte importante de estudo. 


\section{Considerações finais}

A discussão apresentada neste artigo buscou entender, por meio de uma RSL, de que forma os recursos multimídia de RA estão sendo utilizados para o aprimoramento das experiências em laboratórios remotos, bem como os avanços e lacunas do estado da arte. Dentre os avanços, pode-se destacar o aumento de produções com o passar do tempo, a exploração dos tipos de experimentos que podem ser desenvolvidos a partir dos recursos de RA com laboratórios remotos, as tecnologias utilizadas para tal desenvolvimento e a quantidade de trabalhos que focam no nível superior de ensino, principalmente em graduações que envolvem as engenharias e automação.

Como lacunas, pode-se observar a carência de trabalhos de cunho pedagógico que explorem abordagens e estratégias didáticas para o uso dessas tecnologias no contexto de sala de aula, bem como a validação desses recursos junto a professores e alunos. Outra lacuna evidenciada é a ausência de estudos realizados por pesquisadores brasileiros, apesar dos LR já representarem uma tecnologia conhecida na literatura nacional (Da Silva et al., 2016; Lima et al., 2016). Diante disso, faz-se necessário a realização de estudos no Brasil que explorem a RA em laboratórios remotos, com enfoque pedagógica e, principalmente, que considerem a realidade das IES do país.

Por fim, destaca-se o atual cenário da pandemia do COVID-19, do qual IES de todo o mundo estão sob o desafio de continuar ensinando enquanto mantém seus professores, funcionários e alunos a salvo de uma pandemia sem precedente. A solução para muitas instituições foi cancelar as aulas presenciais, incluindo laboratórios e outras experiências de aprendizagem, e determinar que o corpo docente movesse seus cursos para o formato on-line (Hodges, et al). Com isso, recursos tecnológicos inovadores como os apresentados nesse documento, se tornam fundamentais para uma educação on-line eficaz.

Fonte financiadora: Conselho Nacional de Desenvolvimento Científico e Tecnológico CNPq.

\section{Referências}

ANDÚJAR, J. M.; MEJIAS, A.; MARQUEZ, M. A. Augmented reality for the improvement of remote laboratories: An augmented remote laboratory. IEEE Transactions on Education, v. 54, n. 3, p. $492-$ 500, 2011.

ARICI, F.; YILDIRIM, P.; CALIKLAR, S..; YILMAZ, R. M. Research trends in the use of augmented reality in science education: Content and bibliometric mapping analysis. Computers \& Education, v. 142, p. 103647, 2019.

AZUMA, R. T. A survey of augmented reality. Presence: Teleoperators \& Virtual Environments, v. 6, n. 4, p. 355-385, 1997.

BANKY, G. P.; BLICBLAU, A. S. Mimicking of face-to-face experimental venue affordances in an online real-time supervised remote experimental learning context. Global Journal of Engineering Education, v. 21, n. 3, 2019.

CARDOSO, A.; RESTIVO, M. T.; QUINTAS, M. R.; CHOUZAL, F.; RASTEIRO, M. G.; MARQUES, J. C.; MENEZES, P. Online experimentation: Experiment@Portugal 2012. Proceedings of 2014 11th International Conference on Remote Engineering and Virtual Instrumentation, REV 2014, 2014. Conference Paper. p.303-308.

CASSINI, M.; PRATTICHIZO, D. E-Learning by Remote Laboratories: a new tool for controle education. The 6th IFAC Conference on Advances in Control Education, Finland, 2003.

CHACZKO, Z.; ALENAZY, W.; CARRION, L.; TRAN, A. Augmented Reality based monitoring of the remote-lab. ITHET 2014 - 13th International Conference on Information Technology Based Higher Education and Training, 2014. Conference Paper.

COCHRANE. Cochrane Handbook for Systematic Reviews of Interventions. 2019. Disponível em: < https://training.cochrane.org/handbook $>$. 
DA SILVA, J. B.; SIMÃO, J. P. S.; DA SILVA CRISTIANO, M. A.; NICOLETE, P. C.; HECK, C.; DOS SANTOS COELHO, K. A DC Electric Panel Remote Lab. International Journal of Online Engineering (iJOE), v. 12, n. 04, p. 30-32, 2016.

DOS SANTOS, A. C.; FERNANDES, F. S.; DA SILVA, J. B. O uso de laboratórios online no ensino de ciências: uma revisão sistemática da literatura. ScientiaTec, v. 4, n. 1, p. 143-159, 2017.

FABREGAS, E.; DORMIDO-CANTO, S.; DORMIDO, S. Virtual and Remote Laboratory with the Ball and Plate System. IFAC-PapersOnLine, v. 50, n. 1, p. 9132-9137, 2017.

GRANADO, E.; ABAD, J. Z.; PALOMEQUE, F. Q. An approach to develop a LabVIEW based augmented reality application for smartphones. IECON Proceedings (Industrial Electronics Conference), 2016. Conference Paper. p.4970-4975.

GRODOTZKI, J.; ORTELT, T. R.; TEKKAYA, A. E. Remote and Virtual Labs for Engineering Education 4.0: Achievements of the ELLI project at the TU Dortmund University. Procedia Manufacturing, v. 26, p. 1349-1360, 2018.

GUENAGA, M.; MENCHACA, I.; DE GUINEA, A. O.; DZIABENKO, O.; GARCÍA-ZUBÍA, J.; SALAZAR, M. Serious games, remote laboratories and augmented reality to develop and assess programming skills. Lecture Notes in Computer Science (including subseries Lecture Notes in Artificial Intelligence and Lecture Notes in Bioinformatics). 8264 LNCS: 29-36 p. 2014.

HERPICH, F. Recursos educacionais em realidade aumentada para o desenvolvimento da habilidade de visualização espacial em física. 2019.

HERPICH, F.; GUARESE, R. L. M.; TAROUCO, L. M. R. A comparative analysis of augmented reality frameworks aimed at the development of educational applications. Creative Education, v. 8, n. 09, p. $1433,2017$.

LASICA, I. E.; KATZIS, K.; MELETIOU-MAVROTHERIS, M.; DIMOPOULOS, C. Research challenges in future laboratory-based STEM education. Bulletin of the Technical Committee on Learning Technology, v. 18, n. 1, p. 2-5, 2016.

LASICA, I. E.; KATZIS, K.; MELETIOU-MAVROTHERIS, M.; DIMOPOULOS, C. Augmented reality in laboratory-based education: Could it change the way students decide about their future studies? , IEEE Global Engineering Education Conference, EDUCON, 2017. Conference Paper. p.1473-1476.

LIMA, J. P. C.; SIMÃO, J. P. S.; SILVA, I. N.; NICOLETE, P. C.; SILVA, J. B.; ALVES, J. B. M. An inclined plane remote lab. 2016 13th International Conference on Remote Engineering and Virtual Instrumentation (REV), 2016. IEEE. p.7-9.

LO, D. C.-T.; QIAN, K.; QUAN, G.; HONG, L. Work in progress: Enhance CS/CE student learning in computer architecture and organization through a remote instrument control lab with mixed reality. 2012 Frontiers in Education Conference Proceedings, 2012. IEEE. p.1-2.

MAITI, A.; KIST, A.; SMITH, M. Key aspects of integrating augmented reality tools into peer-to-peer remote laboratory user interfaces. Proceedings of 2016 13th International Conference on Remote Engineering and Virtual Instrumentation, REV 2016, 2016. Conference Paper. p.16-23.

MAITI, A.; MAXWELL, A. D.; KIST, A. A. Using marker based augmented reality and natural user interface for interactive remote experiments. Proceedings of 2017 4th Experiment at International Conference: Online Experimentation, exp.at 2017, 2017. Conference Paper. p.159-164.

MARÍN, R.; SANZ, P. J.; NEBOT, P.; WIRZ, R. A multimodal interface to control a robot arm via the web: A case study on remote programming. IEEE Transactions on Industrial Electronics, v. 52, n. 6, p. 1506-1520, 2005.

MÁRQUEZ, M.; MEJÍAS, A.; HERRERA, R.; ANDÚJAR, J. M. Programming and testing a PLC to control a scalable industrial plant in remote way. Proceedings of 2017 4th Experiment at International Conference: Online Experimentation, exp.at 2017, 2017. Conference Paper. p.105-106.

MARTÍN-GUTIÉRREZ, J.; FABIANI, P.; BENESOVA, W.; MENESES, M. D.; MORA, C. E. Augmented reality to promote collaborative and autonomous learning in higher education. Computers in Human Behavior, v. 51, p. 752-761, 2015.

MEJÍAS, A.; ANDÚJAR, J. M. Interaction of real robots with virtual scenarios through augmented reality: Application to robotics teaching/learning by means of remote labs. International Journal of Engineering Education, v. 29, n. 3, p. 788-798, 2013.

MEJÍAS, A.; SÁNCHEZ, M. A. M.; ANDÚJAR, J. M.; HERRERA, M. R. S. A complete solution for developing remote labs. IFAC Proceedings Volumes (IFAC-PapersOnline), 2013. Conference Paper PART 1. p.96-101. 
MEJÍAS, A. B.; ANDÚJAR, J. M. A Pilot Study of the Effectiveness of Augmented Reality to Enhance the Use of Remote Labs in Electrical Engineering Education. Journal of science education and technology, v. 21, n. 5, p. 540-557, 2012.

ODEH, S.; SHANAB, S.; ANABTAWI, M. Augmented Reality Internet Labs versus its Traditional and Virtual Equivalence. International Journal of Emerging Technologies in Learning, v. 10, n. 3, 2015. ODEH, S.; SHANAB, S.; ANABTAWI, M.; HODROB, R. Remote augmented reality engineering labs. IEEE Global Engineering Education Conference, EDUCON, 2012. Conference Paper.

ODEH, S; SHANAB, S; ANABTAWI, M; HODROB, R. A remote engineering lab based on augmented reality for teaching electronics. International Journal of Online Engineering, v. 9, p. 61-67, 2013.

RODRIGUES, J.; ANDRADE, T.; ABREU, P.; RESTIVO, M. T. Adding augmented reality to laboratory experimentation. Proceedings of 2017 4th Experiment at International Conference: Online Experimentation, exp.at 2017, 2017. Conference Paper. p.135-136.

RODRIGUEZ-GIL, L; ZUBIA, J; ORDUÑA, P; LOPEZ-DE-IPINA, D. Towards new multiplatform hybrid online laboratory models. IEEE Transactions on Learning Technologies, v. 10, n.3, p.318-330, 2017.

SAHIN, D.; YILMAZ, R. M. The effect of Augmented Reality Technology on middle school students' achievements and attitudes towards science education. Computers \& Education, v. 144, p.103710, 2020. SALZMANN, C.; GILLET, D.; HUGUENIN, P. Remote experimentation: Improving user perception using augmented reality 2000.

SHANAB, S. A.; ODEH, S.; HODROB, R.; ANABTAWI, M. Augmented reality internet labs versus hands-on and virtual labs: A comparative study. Interactive Mobile and Computer Aided Learning (IMCL), 2012 International Conference on, 2012. IEEE. p.17-21.

SMITH, M.; MAITI, A.; MAXWELL, A.; KIST, A. A. Applying Augmented Reality to New or Existing Remote Access Laboratories. 2019 5th Experiment International Conference, 2019. IEEE. p.6-11.

SMITH, M.; MAITI, A.; MAXWELL, A. D.; KIST, A. A. Augmented and mixed reality features and tools for remote laboratory experiment. International Journal of Online Engineering, v. 12, n. 7, p. 4552, 2016 .

SMITH, M; MAITI, A; MAXWELL, A; KIST, A. Augmented and mixed reality features and tools for remote laboratory experiments. International Journal of Online Engineering, v.12, n.7, p.45-52, $2016 \mathrm{~b}$. SUN, H.; ZHANG, Z.; LIU, Y.; DUH, H. B. L. OptoBridge: Assisting skill acquisition in the remote experimental collaboration. Proceedings of the 28th Australian Computer-Human Interaction Conference, OzCHI 2016, 2016. Conference Paper. p.195-199.

VARGAS, H.; FARIAS, G.; SANCHEZ, J.; DORMIDO, S.; ESQUEMBRE, F. Using augmented reality in remote laboratories. International Journal of Computers, Communications and Control, v. 8, n. 4, p. 622-634, 2013.

ZANDAVI, S. M.; CHUNG, V. Augmented Reality for Remote Laboratory Improving Educational Learning: Using Elevated Particle Swarm Optimization in Object Tracking Scheme. 2018 International Joint Conference on Neural Networks (IJCNN), 2018. IEEE. p.1-6. 